\title{
Tideglusib: The Miracle Molecule for Tooth Repair
}

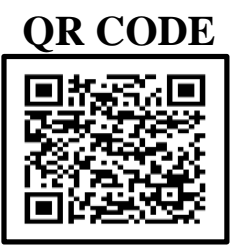

Dental caries is the most common reason behind a dental visit. The prevalence of dental caries across the globe is too high and restoration, commonly known as filling is the conventional treatment to repair damage caused by tooth decay wherein the procedure typically involves removing the damaged part of the tooth and replacing it with a restorative material which stays in the tooth and does not degrade. Thus, the normal mineral volume of the tooth is never restored completely. At late stages, when pulp is involved, time intensive root canal treatment is done. For teeth that cannot be saved by these treatment options, extraction is the only answer. The revolutions in biological and digital information are quickly converging with dental science and dentistry is at the threshold of new discoveries. ${ }^{1}$

Glycogen synthase kinase-3 inhibitor have been studied as a potential therapeutic agents in depression, anxiety, schizophrenia, Alzheimer's disease, supranuclear palsy, fragile $\mathrm{X}$ syndrome, multiple sclerosis, Parkinson's disease, Huntington's disease, stroke, traumatic brain injury, spinocerebellar ataxia type 1 , sepsis, asthma, arthritis, colitis, peritonitis and various types of cancers. ${ }^{2}$ These drugs have the capacity to modulate human stem cells in vivo.

Tideglusib, a glycogen synthase kinase- 3 inhibitor, initially tested for its role in Alzheimer's disease and psychiatric disorders, has the potential to change the conventional treatment of dental caries owing to its property to regrow dentine to fill a void. Research suggests that this drug could help protect the inner layer by stimulating the production of the dentine allowing the tooth to repair itself. Zaugg LK et al., in their study on rat teeth, showed that Tideglusib can equally enhance reparative dentine formation to fully repair an area of dentine damage up to 10 times larger, mimicking the size of small lesions in human. In this research, Tideglusib treated teeth revealed significantly more mineralized tissue and more dentine volume compared to the control group. The researchers were able to repair a surface damage area of $0.1 \mathrm{~mm}^{2}$ and volume of $0.055 \mathrm{~mm}^{3}$ completely. ${ }^{3}$ The mechanism behind this act of Tideglusib seems to be the activation of the $\mathrm{Wnt} / \beta$-cat signaling pathway of resident mesenchymal stem cells from the tooth pulp wherein Axin 2 is a negative regulator and a downstream target. The enzyme glycogen synthase kinase 3 is a prime cytoplasmic component of $\mathrm{Wnt} / \beta$ -cat signal transduction that phosphorylates Beta catenin and Axin, furthermore leading to ubiquitination and degradation. This all happens in the absence of Wnt ligand/receptor binding. When these ligands are available, GSK-3 activity is inhibited and this allows Beta-catenin to enter the nucleus where it interacts with Lef/Tcf transcription factors and regulates the expression of the target genes including Axinz. The addition of Wnt signaling agonists provides an effective way to stimulate reparative formation of dentinal tissue and restores lost dentine with naturally generated new dentinal tissue. ${ }^{4}$

The dental tissue produced by Tideglusib stimulation integrates itself completely with the enamel tissue which means the tooth is not only simply repaired and compensated but is regrown and restored back to health. ${ }^{5}$

In a study conducted by del Ser et al., the use of Tideglusib was associated with mild to moderate adverse drug reactions, including transient increase in serum creatine kinase, ALT or gGT, diarrhea, nausea, cough, fatigue, and headache. ${ }^{6}$ Tideglusib is remarkably cheap and has a good safety profile making it more compliant with the subjects.

Regenerative medicine aims to restore the original composition, functions and properties of lost tissues. A novel, biology based new generation treatment for dental caries is required. The use of Tideglusib is such an approach where dentinal restoration is stimulated via the mobilization of resident stem cells in the tooth pulp leading to the formation of natural reparative 
dentine. Promising potential for translational research approaches has been highlighted by use of tideglusib in animal studies. This property needs to be tested in human subjects respecting the ethical and regulatory criteria. Large scale clinical trial to explore the efficacy and safety of tideglusib in dental practice need to be designed and executed.

\section{REFERENCES}

1. Seldin LW, Committee FT, American Dental Association Future of Dentistry Report. The future of dentistry: An overview of a new report. The Journal of the American Dental Association 2001; 132(12):166777.

2. Hostiuc S, Perlea P, Marinescu M, Dogaroiu C, Drima E. GSK-3 Inhibitors and Tooth Repair: An Ethical Analysis. Front Pharmacol. 2019;9:1495. https://doi.org/10.3389/fphar.2018.01495 3. Zaugg LK, Banu A, Walther AR, Chandrasekaran D, Babb RC, Salzlechner C et al. Translation Approach for Dentine Regeneration Using GSK-3 Antagonists. J
Dent Res. 2020;99(5):544-51. https://doi.org/10.1177/oo22034520908593

4. Neves VCM, Babb R ,Chandrasekaran D, Sharpe PT. Promotion of natural tooth repair by small molecule GSK3 antagonists. Sci Rep. 2017; 7: 39654. https://doi.org/10.1038/srep39654 5. Ricci WA, Lucas CD, Piveta AC, Nagle MM, Montandon AA. Clinical application of adhesive systems-a critical review: biomimetic approach. RGO-Revista Gaúcha de Odontologia. 2015; 63(1):55-62. https://doi.org/10.1590/1981863720150001000082489

6. del Ser T, Steinwachs KC, Gertz HJ, Andrés MV, Gómez-Carrillo B, Medina $\mathrm{M}$ et al. Treatment of Alzheimer's disease with the GSK-3 inhibitor tideglusib: a pilot study. J Alzheimers Dis. 2013;33(1):205-15. https://doi.org/10.3233/JAD-2012120805.

Cite this article as:

Gupta S. Tideglusib: The Miracle Molecule for Tooth Repair. Int Healthc Res J. 2020;4(9):GC1-GC2. https://doi.org/10.26440/IHRJ/0409.12307 